\title{
Augmenting Resource Allocation Techniques for the Management of ICU Beds During COVID-19 Pandemic
}

\author{
Seyed Mohssen Ghafari, Richard Nichol, and Richard A. George \\ Faethm AI Company, Sydney, Australia
}

\begin{abstract}
At the time of writing, more than seventy million people have been infected by COVID19 and more than one and a half million have died from the infection. A major challenge for health systems around the world is to supply ventilators and Intensive Care Unit (ICU) beds for those patients with the most severe symptoms of the infection. Unfortunately, during the COVID-19 pandemic, many countries face ICU bed shortages. In situations of peak-demand, healthcare providers follow predefined strategies to allocate the available ICU beds in the most efficient way. On these occasions, physicians and healthcare workers, who swore an oath to treat the ill to the best of their ability, would have to choose not to save some patients to ensure others survive. This decision puts healthcare professionals in an ethically and emotionally challenging situation in an already stressful environment. In this paper, we propose an automatic approach for managing ICU beds in hospitals to i) create the most effective ICU resource allocation, and ii) relieve physicians of having to make decisions in this regard. The experimental results demonstrate the effectiveness of our approach.
\end{abstract}

Keywords: COVID-19 · Resource Allocation · ICU Beds · Regression.

\section{Introduction}

More than 70 million people have been infected by the newly discovered Coronavirus (COVID-19) and more than one and half million have died because of COVID-19. If we consider the world's population as 7.7 billion people ${ }^{1}$, around $0.1 \%$ of world's population has now been infected by this virus ${ }^{2}$.

Over the past few months, because of the dramatic increase in the number of infected people, different countries have faced shortages in Intensive Care Units (ICU) beds: there were not enough ICU beds for those who needed intensive care, leading to deaths that could be avoided with adequate resources and effective use of allocation techniques.

\footnotetext{
${ }^{1}$ https://ourworldindata.org/world-population-growth

2 https://www.cnbc.com/2020/06/24/who-warns-coronavirus-still-hasnt-reached-itspeak-in-americas.html
} 


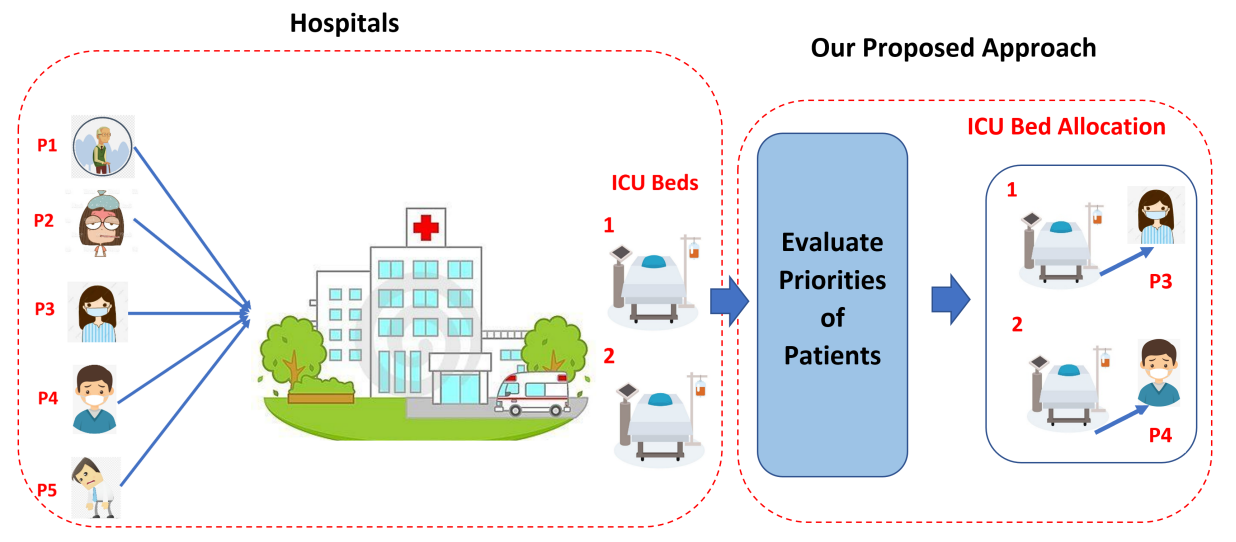

Fig. 1. Our proposed framework in a simple hospital scenario: there are five patients, one hospital, and two ICU beds. Our proposed approach is able to prioritise the patients and allocate the available ICU beds to the patients with the highest priority. For instance, in this scenario, patients 3 and 4 have the highest priority.

Health systems all over the world follow predefined regulations to distribute ICU beds to people based on different factors to determine whether an individual should be saved or not. To the best of our knowledge, the decisions on how to distribute ICU beds among patients are made by health workers and physicians. Hence, as it is expected, any manual human-based process i) could be unfair and be a decision based on emotion rather than logic ii) put unnecessary burden (both emotionally and ethically) on the decision maker.

In this paper, we propose an automatic ICU beds allocation model. We assume that ICU beds are resources and we can apply CPU (Central Processing Unit)-allocation algorithms to manage them. We collected data from different health systems around the world, and we present the different key criteria that may affect the priority of a patient to receive an ICU bed. Next, using those key criteria, we define the priorities of patients. Finally, with employing a resource allocation algorithm, we propose an ICU bed allocation algorithm useful for pandemics and other peak demand scenarios. The contributions of this paper are as follows:

- To the best of our knowledge, this is the first automatic ICU bed allocation algorithm that could be used in the case of a pandemic.

- We present the key criteria that may affect the priority of patients for receiving ICU beds.

- The experimental results demonstrate the effectiveness of our proposed approach.

The rest of the paper can be organised as follows: We present the process of defining a priority for a patient and our ICU beds allocation approach in Section 2. The experimental results are presented in Section 3, and our conclusion is in Section 4. 
Table 1. The process of calculating Sequential Organ Failure Assessment (SOFA) Score

\begin{tabular}{||c|c|c|c|c|c|c||}
\hline Variable & 0 & 1 & 2 & 3 & 4 & Score $(0-4)$ \\
\hline \hline Level of oxygen in blood & $>400$ & $<400$ & $<300$ & $<200$ & $<100$ & \\
\hline Platelets $^{3}$ & $>150$ & $<150$ & $<100$ & $<50$ & $<20$ & \\
\hline Liver function (Bilirubin) & $<1.2$ & $1.2-1.9$ & $2.0-5.9$ & $6.0-11.9$ & $>12$ & \\
\hline Low blood pressure (Hypotension) & None & MABP $<70 \mathrm{mmHg}$ & Dop $<5$ & Dop 6-15 & DOP $>15$ & \\
\hline Neurologic function & 15 & $13-14$ & $10-12$ & $6-9$ & $<6$ & \\
\hline Kidney function (Creatinine) & $<1.2$ & $1.2-1.9$ & $2.0-3.4$ & $3.5-4.9$ & $>5$ & \\
\hline
\end{tabular}

\section{ICU Beds Management}

In this section, we discuss our method to calculate a priority score; how we allocate ICU beds to those patients; propose a survival rate factor; and provide an example to show how our approach works in a potential scenario.

\subsection{Patient Priority}

In this subsection, we discuss the main criteria that different health systems consider for prioritising their patients and we present a formula to define patients' priorities. The general guidelines of health systems around the world for managing ICU beds and ventilators suggest to focus on saving larger number of patients and save those that have more potential years of life ${ }^{4}$. Moreover, in some countries, like the US, the priorities is given to homeless people, since they do not have any place to safely self-quarantine and recover, and healthcare workers, because they are valuable for health systems.

In this paper, in addition to considering the above mentioned factors, we also consider the mortality risk assessment factor suggested in ventilator allocation guidelines (New York state task force on life and the law New York state department of health ${ }^{5}$ ). According to this guideline, the patient's mortality risk can be assessed by a clinical scoring system, i.e, Sequential Oral Failure Assessment (SOFA). According to the guideline, SOFA can be assessed by Table 1. In this table, each variable will be assigned by a score between zero (best score) and four (worst score). A total score of 24 indicates a life threatening situation. According to this guideline, "the more severe a patient's health condition (i.e., higher the $S O F A$ score) and worsening/no change in mortality risk (i.e., increase or little/no change in the SOFA score), the less likely the patient continues with ventilator therapy." The patients' status will be monitored regularly during the 48 and 120 hours after allocating a ventilator to them to check their

\footnotetext{
${ }^{4}$ https://www.nytimes.com/2020/03/12/world/europe/12italy-coronavirus-healthcare.html

${ }^{5}$ https : //www.health.ny.gov/regulations/task $k_{f}$ orce/
} 
SOF A score. Having the above mentioned factors, now we are able to propose a regression based [6][7] priority assessment mechanism for prioritising patients as follows:

$$
\text { Priority }_{i t}=w_{1} \times P L_{i}+w_{2} \times H W_{i}+w_{3} \times H L i-w_{4} \times S O F A_{i},
$$

where Priority $_{i t}$ is the priority of $i^{\text {th }}$ patient to receive an ICU bed and ventilator in the time $t$; $P L_{i}$ indicates the potential years of life of $i^{t h}$ patient and this could be different in various countries based on the difference between age of patients and average life expectancy in those countries. $H W_{i}$ denotes whether $i^{\text {th }}$ patient is a healthcare worker (1) or not $(0), H L_{i}$ is 1 if $i^{\text {th }}$ patient is homeless and 0 if he/she is not, and $S O F A_{i}$ represents the SOFA score of $i^{t h}$ patient. In this formula, $w_{1}, w_{2}, w_{3}$, and $w_{4}$ are controlling parameters to control the effects of our considered criteria. Finally, $w_{4} \times S O F A_{i}$ has a negative sign in this formula to ensure a negative affect if $i^{\text {th }}$ patient has a higher $S O F A$ (a severe health condition).

Since $P L_{i}$ and $S O F A_{i}$ could be larger numbers than $H W_{i}$ and $H L_{i}$, we normalise them to the range between 0 and 1 by a feature scaling approach [4]. For instance, for $P L_{i}$ we have:

$$
P L_{i}^{\prime}=\frac{P L_{i}-\min (P L)}{\max (P L)-\min (P L)}
$$

\subsection{CPU Scheduling Algorithms}

In this subsection, we present our proposed approach for ICU beds allocation during a pandemic, e.g, COVID-19. We propose to use CPU scheduling algorithms to manage ICU beds and consider a bed as a CPU resource. Generally, there are two types of CPU scheduling algorithms: Preemptive and NonPreemptive scheduling.

Preemptive scheduling algorithms allocate $\mathrm{CPU}$ resources to the processes for a limited amount of time with a condition: if a processes with a higher priority arrive in the waiting queue (a queue of processes which waiting for receiving CPU resources), the allocated CPU resources will be taken away to be allocated to the recent arrived high priority process. Algorithms like Round Robin [1], Shortest Remaining Time First [1], preemptive version of Priority algorithm belong to this category of CPU scheduling algorithms. However, in non-Preemptive scheduling algorithms, an allocated CPU resource may not be taken away from a process even if a process with a higher priority arrives in the waiting queue. Shortest Job First and non-preemptive version of Priority algorithm are examples of non-Preemptive CPU scheduling algorithms.

According to the mentioned ventilator allocation guidelines from New York state task force, "at any point during the time trial, even before an official assessment occurs, if a patient develops a condition on the exclusion criteria list and there is an eligible patient waiting, then the ventilator is reallocated". 
Table 2. Example Scenario. A smaller arrival time indicate that patient arrived earlier than others.

\begin{tabular}{||c|c|c|c|c|c|c|c||}
\hline Patient ID & $t_{i}$ & $P L_{i}$ & $H W_{i}$ & $H L_{i}$ & $S O F A_{i}$ & Required ICU Beds Occupancy Time & Discharge Time \\
\hline \hline 1 & 0 & 20 & 0 & 0 & 15 & 21 & - \\
\hline 2 & 1 & 25 & 0 & 0 & 18 & 18 & - \\
\hline 3 & 1 & 45 & 1 & 0 & 10 & 10 & 10 \\
\hline 4 & 10 & 50 & 0 & 1 & 10 & 14 & 26 \\
\hline
\end{tabular}

Hence, for managing ICU beds and ventilators, we need the same approach as preemptive CPU scheduling algorithms. In this paper, we use the terms CPU resources and ICU beds and processes and patients interchangeably.

\subsection{Scheduling Approach}

The toughest decision for healthcare worker is not only on how they should distribute the ICU beds, but also taking back an ICU bed from a patient if another patient with a higher priority presents. Sadly, this is a rule in many of the health systems around the world to save the most valuable lives. This is a very good indication that these ICU beds should be scheduled by preemptive resource scheduling techniques. Hence, in this paper, we also follow the same approach for our ICU bed scheduling process.

The preemptive scheduling algorithm that we employ for managing ICU beds is preemptive priority scheduling [5]. According to this algorithm, the time of receiving ICU beds for patients is not only based on resources burst time, but also it is based on the priority of each patient, i.e., high priority patients receive ICU beds earlier than others. In this regard, the patients with the same priority will be served as first come first serve manner. In the rare case of having patients with the same priority and the same arriving time, we also follow the New York health guideline mentioned in Section $2^{6}$ : if these patients are adults and if the number of ICU beds are less than the number of patients, we use a random process (e.g., lottery) to allocate ICU beds to these patients.

\subsection{Survival Rate Calculation}

In this subsection, we propose a survival rate factor that could be used to evaluate an ICU bed allocation mechanism from the point of view of the number of saved patients. In this paper, we assume if a patient presents in a hospital and he/she requires an ICU bed for his/her treatments, the maximum waiting time for him/her would be one day, otherwise he/she would be transferred to another hospital or sadly, he/she will die. Hence, we may evaluate the performance of an ICU bed allocation model with respect to its survival rate that indicates

\footnotetext{
${ }^{6}$ https : //www.health.ny.gov/regulations/task ${ }_{f}$ orce/
} 
the number of high priority patients that it could save within a day after their present in the hospital. We propose the following formula for calculating the survival rate:

$$
S R=\left(\frac{H P}{T H P}\right) * 100
$$

where $S R$ represents the survival rate of an ICU bed allocation mechanism, $H P$ denotes the number of high priority patients that were served with an ICU bed within one day of their presentation in the hospital; and $T H P$ indicates the total number of high priority patients that are presented in that hospital. Obviously, a higher $S R$ demonstrates the effectiveness of an ICU bed allocation approach.

\subsection{An Example Scenario}

Assume we have a hospital with one ICU bed. Four patients with serious health conditions present to the hospital. In this scenario, $t_{i}$ represents the arrival time of patients, where $i=\{1,2,3,4\}$. Based on the result of medical examination of these patients, we can assess their SOFA score (reported in Table 2).

In Table 2, each patient has a required ICU bed occupancy time. First of all, this occupancy time is an estimation time and we only mention that here to simplify our example. However, in a real-world scenarios, providing this estimation time may be hard and sometimes impossible for many of the patients. Based on this scenario, our patients arrive at times $0,1,1$, and 10, respectively. Our approach first assess the priority of each patient based on Formula 1. In this example scenario, we assume all the controlling parameters are equal to each other and their value is 0.25 .

In time 0 , we have only one patient (patient 1 ). Since, there is one ICU bed available and there is not any other patients in the hospital, the ICU bed will be allocated to him/her without assessing his/her priority. However, in time 1, we have three patients. The ICU bed is already allocated to patient 1, but the challenging step is to assess the priority of these patients for possible reallocation of the ICU bed to the two new arrived patients. $P L_{i}^{\prime}$ is $0,0.33,0.83$ for patients 1,2 , and 3 , respectively (according to Formula 2). Using the same feature scaling formula for SOFA, the SOFA scores are $0.62,1$, and 0.25 for patients 1,2 and 3 , respectively. According to the Formula 1, the priorities are $0.15,-0.16$, and 0.39 for patients 1,2 and 3 , respectively. As a result, the allocated ICU bed will be taken away from patient 1 and will be reallocated to the patient 3 , as this patient has a higher priority than patients 1 and 2 .

The health status of the patient 3 will be monitored, his/her priority score will be recalculated over time and be compared against the priority of other patients. In this example, we assume the SOFA score remains the same for patients over the time. Hence, until the time 10 there are no other patient with a higher priority than patient 3 and the ICU bed will serve him/her until this time and until he/she is discharged from the hospital. At time 10, a new patient arrives (patient 4). The priority of this patient is 0.5. Although patients 1 and 2 are waiting for the ICU bed, the free ICU bed will be allocated to patient 4 


\begin{tabular}{|c|c|c|c|c|c|c|c|}
\hline & Process_ID & Arrival_Time & Priority & Orig_Burst_Time & Completion_Time & Turnaround_Time & Waiting_Time \\
\hline 0 & 75 & 2 & 16.125 & 2 & 4 & 2 & 0 \\
\hline 1 & 90 & 8 & 18.375 & 2 & 10 & 8 & 0 \\
\hline 2 & 86 & 3 & 14.125 & 5 & 11 & 6 & 3 \\
\hline 3 & 23 & 12 & 18.125 & 2 & 14 & 12 & 0 \\
\hline 4 & 12 & 14 & 19.625 & 7 & 21 & 14 & 0 \\
\hline 5 & 34 & 15 & 15.875 & 4 & 25 & 21 & 6 \\
\hline 6 & 100 & 15 & 14.875 & 2 & 27 & 25 & 10 \\
\hline 7 & 32 & 20 & 13.875 & 2 & 29 & 27 & 7 \\
\hline 8 & 5 & 0 & 13.375 & 6 & 32 & 26 & 26 \\
\hline 9 & 59 & 9 & 13.125 & 5 & 37 & 32 & 23 \\
\hline 10 & 83 & 9 & 13.125 & 4 & 41 & 37 & 28 \\
\hline 11 & 88 & 15 & 13.125 & 5 & 46 & 41 & 26 \\
\hline 12 & 55 & 19 & 12.625 & 6 & 52 & 46 & 27 \\
\hline 13 & 97 & 5 & 12.375 & 7 & 59 & 52 & 47 \\
\hline 14 & 67 & 4 & 11.625 & 4 & 63 & 59 & 55 \\
\hline
\end{tabular}

Fig. 2. ICU bed services for the top 15 priority Patients using our proposed model.

(since he/she has a higher priority). Until time 26, patient 4 continue to use the ICU bed and will be discharged from the hospital at this time.

What happens to the patients 1 and 2? At time 26, either they were referred to another hospital, or unfortunately they did not succeed in receiving any ICU services. However, the decision to not save them is not from a healthcare worker. This decision is based on an automatic approach, and it is based on a fair and unemotional mechanism (similar to Association Rule Mining (ARM) techniques [8]).

\section{Experiments}

Here, we test our proposed method on a sample dataset.

\subsection{Experimental Setup}

A sample dataset with 100 patients is created to test the model. For each patient, we randomly initialized the arriving time and values of factors mentioned in Section 2.1. Next, we calculated the priority of each patient based on Formula 1. Then, for calculating the potential years of life, we calculated the difference between the average life expectancy in Australia $\left(82.50{ }^{7}\right.$ ) and the age of each

\footnotetext{
$\overline{7}$ https://www.worldbank.org/
} 


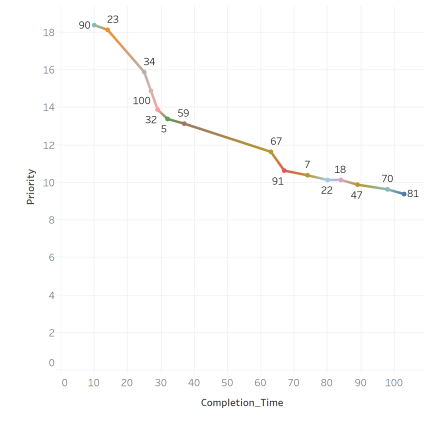

Fig. 3. ICU bed services for the top 15 priority Patients

Table 3. An Example Scenario. A smaller arrival time indicate that patient arrived earlier than others. AWT and HPP are abbreviations for Average Waiting Time and High Priority Patients.

\begin{tabular}{||c|c|c|c|c|}
\hline Method & AWT For All Patients & Treated HPP & AWT For HPP & SR \\
\hline \hline Our Approach & 215.5 & 15 & 17.2 & $27 \%$ \\
\hline Shortest Job First Algorithm [1] & 135.88 & 1 & 146.5 & $6 \%$ \\
\hline First Job First Serve Algorithm [1] & 189.2 & 2 & 194.6 & $0 \%$ \\
\hline
\end{tabular}

patient. We selected Australia as an example, and this approach could be applied on any other countries as well. Moreover, in this paper, we assume all the controlling parameters in Formula 1 are equal to 0.25 [3] [2] and leave optimizing these weights with a proper optimization algorithm for our future work. In addition, although our proposed method is capable for a dynamic situation and deal with unknown required ICU bed occupancy time, in this paper, to simplify our experiments, we assume a predefined time, which is a random number between one and seven, for each patient as their required ICU bed occupancy time. Finally, We assume we have only one available ICU bed.

\subsection{Experimental Results}

Figure 2 illustrates the ICU bed services for top 15 high priority patients. In this table, Process-ID, Arrival-Time, Orig-Burst-Time, Completion-Time, Turnaround-Time, and Waiting-Time are indicating patient id, arrival time to the hospital, the required ICU bed occupancy time, the discharge time, the time that the patient received the ICU bed, and the time that the patient was waiting for receiving an ICU bed.

According to Figure 2, our proposed method is capable of automatically managing the patients based on their priorities. The reason behind the large waiting time for some of the patients is that we assumed we only have one ICU bed. In many of the hospitals around the world, there are several ICU beds that can 
be allocated to the patients, leading to reducing the waiting time, significantly. Moreover, Figure 3 illustrates the completion time for some of the high priority patients in our experiment. The higher priority patients have smaller completion time indicating that our proposed model focuses more on saving patients with higher priority.

In this paper, we also compare the performance of our proposed approach with other CPU schedulers, i.e., shortest job first (SJF) and First Job First Serve (FJFS) algorithms, with respect to average waiting time to be served for all patients, the number of treated high priority patients, average waiting time to be served for high priority patients, and SR (survival rate). In this section, the term high priority refers to the top-15 patients that have the highest priority among the all patients in our experiment (reported in Figure 2), and the term 'Treated High Priority Patients' indicates how many of these high priority patients were among the top- 15 first serve patients by the mentioned algorithms, which can demonstrate the emphasise that an algorithm has on saving high priority patients. The comparison results are reported in Table 3. According to this table, although our proposed approach has the highest average waiting time compared to SJF and FJFS algorithms, it first saves 15 out of 15 of the top-15 high priority patients which this number is 1 and 2 for SJF and FJFS, respectively.

Moreover, in our proposed approach, the average waiting time for the top15 high priority patients is 17.2 , while this number is 146.5 and 194.6 for SJF and FJFS, respectively. As our intention was to serve the high priority patients earlier, the results reported in Table 3 indicates we are succeeded in achieving this goal. It is worth mentioning that in any other on-demand scenarios, we can change the priority criteria and consider other kind of factors, e.g., first saving children or pregnant women.

Finally, we may compare our proposed approach with SJF and FJFS with respect to SR. Table 3 demonstrates that our proposed approach has the highest survival rate for the top- 15 high priority patients and could serve $27 \%$ of them within one day of their presentation in a hospital. It is worth mentioning that this number is for a situation that we have only one ICU bed for 100 patients and increasing the number of available ICU beds may increase SR of our proposed approach, significantly.

\section{Technology Ethics}

In this section, we discuss the ethical concerns related to adopting our proposed approach in a real-world health system. Just like adopting any other technology, using the proposed ICU bed allocation approach in a real-world scenario requires an extensive risk management process. As we are at the early stage of this line of research, we strongly advise people to assess the existential risk of adopting our proposed approach or any similar algorithm before practically employ that in the health systems. 


\section{Conclusion and Future Work}

In this paper, we proposed an automatic ICU bed allocation method, which can be used in a Pandemic or any other high demand situation. The goal of this method is to make a fair ICU bed distribution among patients and relieve health workers and physicians from making tough decisions to stop saving someone because of ICU bed shortages in hospitals during a pandemic or a high demand situation. We discuss an example scenario to further explain our proposed approach, and finally, tested it on a sample dataset of 100 patients. For our future direction, we will focus on improving this approach by employing more sophisticated methods to deal with large volumes of data, e.g., deep neural network-based algorithms. Further, we plan to test our approach on real-world hospital data to compare effectiveness of an automatic approach with a manual and human-based mechanism. And at last, we also tend to extend this method to a distributed ICU bed allocation algorithm which can deal with different ICU beds in different hospitals.

\section{References}

1. Arpaci-Dusseau, R.H., Arpaci-Dusseau, A.C.: Operating Systems: Three Easy Pieces. Arpaci-Dusseau Books (2018)

2. Ghafari, S.M., Yakhchi, S., Beheshti, A., Orgun, M.A.: SETTRUST: social exchange theory based context-aware trust prediction in online social networks. In: Data Quality and Trust in Big Data - 5th International Workshop, QUAT 2018, Held in Conjunction with WISE 2018, Dubai, UAE. Lecture Notes in Computer Science, vol. 11235, pp. 46-61. Springer (2018)

3. Ghafari, S.M., Yakhchi, S., Beheshti, A., Orgun, M.A.: Social context-aware trust prediction: Methods for identifying fake news. In: Web Information Systems Engineering - WISE 2018 - 19th International Conference, Dubai, United Arab Emirates (2018)

4. Juszczak, P., Tax, D., Duin, R.: Feature scaling in support vector data description (2002)

5. Silberschatz, A., Gagne, G., Galvin, P.B.: Operating System Concepts. John Wiley and Sons (2009)

6. Yakhchi, S., Beheshti, A., Ghafari, S.M., Orgun, M.A.: Enabling the analysis of personality aspects in recommender systems. In: 23rd Pacific Asia Conference on Information Systems, PACIS China. p. 143 (2019)

7. Yakhchi, S., Ghafari, S.M., Beheshti, A.: CNR: cross-network recommendation embedding user's personality. In: Data Quality and Trust in Big Data - 5th International Workshop, QUAT 2018, Held in Conjunction with WISE 2018, Dubai, UAE. Lecture Notes in Computer Science, vol. 11235, pp. 62-77. Springer (2018)

8. Yakhchi, S., Ghafari, S.M., Tjortjis, C., Fazeli, M.: Armica-improved: A new approach for association rule mining. In: Knowledge Science, Engineering and Management - 10th International Conference, KSEM 2017, Melbourne, VIC, Australia. Lecture Notes in Computer Science, vol. 10412, pp. 296-306. Springer (2017) 


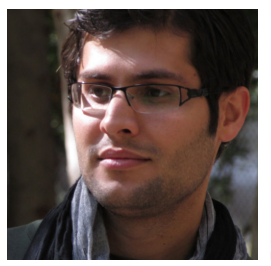

Seyed Mohssen Ghafari. Dr. Seyed Mohssen Ghafari has a $\mathrm{PhD}$ in Computer Science and he is a data scientist at Faethm AI Company. He is interested in proposing solutions for real-world's problems using data science techniques. His research interests are social media analysis, recommender systems, trust prediction, fake news detection and natural language processing. Contact him at seyed-mohssen.ghafari@hdr.mq.edu.au.

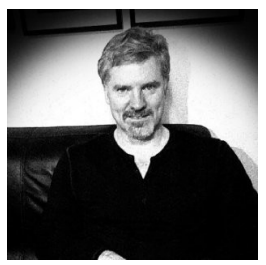

Richard Nichol. Mr. Richard Nichol is the head of Data Science at Faethm AI, with over 20 years of experience working across the ICT, Banking, Insurance, Government, Procurement and Transport sectors. He has a Masters of Data Science from University of Sydney and specializes in Workforce Analytics, Machine Learning, and Natural Language Processing. He is co-author of "Machine Learning for Business" by Manning Publishing. Contact him at richard.nichol@faethm.ai. Contact him at richard.nichol@faethm.ai.

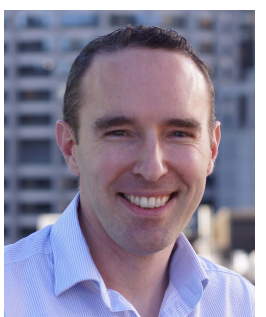

Richard George. Dr. Richard George has a PhD in bioinformatics from University College London and is the Chief Data Scientist at Faethm AI company. His core competencies are predictive analytics, machine learning, artificial intelligence, and business strategy development. Contact him at richard.george@faethm.ai. 